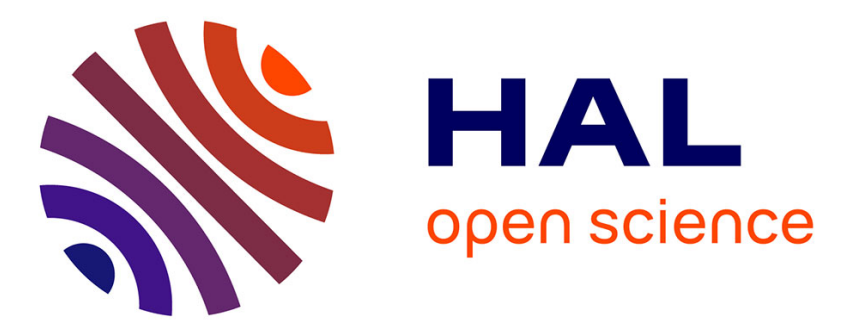

\title{
Développement durable et innovation. Le changement climatique comme levier d'innovation sociale
}

Sylvain Mondon

\section{To cite this version:}

Sylvain Mondon. Développement durable et innovation. Le changement climatique comme levier d'innovation sociale. Prospective et Stratégie, 2016, Innovations, 7 (1), pp.141-155. 10.3917/pstrat.007.0141. halshs-02967391

\section{HAL Id: halshs-02967391 https://shs.hal.science/halshs-02967391}

Submitted on 14 Oct 2020

HAL is a multi-disciplinary open access archive for the deposit and dissemination of scientific research documents, whether they are published or not. The documents may come from teaching and research institutions in France or abroad, or from public or private research centers.
L'archive ouverte pluridisciplinaire HAL, est destinée au dépôt et à la diffusion de documents scientifiques de niveau recherche, publiés ou non, émanant des établissements d'enseignement et de recherche français ou étrangers, des laboratoires publics ou privés. 


\title{
REGARDS D'ACTEURS
}

\section{Développement durable et innovation Le changement climatique comme levier d'innovation sociale}

\author{
Sylvain MONDON
}

La France, par la variété de son territoire métropolitain comme ultramarin, fait face à des défis très variés pour le long terme dont certains interagissent avec le changement climatique. Au-delà des fluctuations inter-annuelles, le climat évolue selon différentes dynamiques de plus ou moins long terme. L'adaptation des activités humaines aux caractéristiques météorologiques d'un lieu paraît une évidence. Cependant, le contexte du changement climatique provoqué par les activités humaines modifie certains éléments dont la prise en compte fait émerger des besoins nouveaux. Longtemps circonscrits au domaine de la recherche en science du climat dans le but de développer les connaissances, ces besoins nouveaux apparaissent progressivement dans de nombreux autres domaines. Ces besoins sont souvent apparus après une prise de conscience de la vulnérabilité des personnes ou des biens lors d'événements météorologiques représentatifs d'un climat moyen plus chaud comme d'intenses vagues de chaleur ${ }^{1}(1947,1983,1994,2003,2006,2015)$, des périodes de sévère sécheresse des sols $(1976,1989,1990,2003,2005,2011)^{2}$ ou de vaste submersion marine $(2010)^{3}$.

Après avoir examiné dans quelle mesure le climat évolue, les grandes différences des changements constatés actuellement par rapport à ceux que la terre a connus à différentes échelles de temps sont soulignées. Ce panorama climatique du passé et du présent est complété par un aperçu des grandes caractéristiques des projections

1 Karine Laaidi, Aymeric Ung, Mathilde Pascal, Pascal Beaudeau, Vulnérabilité à la chaleur : actualisation des connaissances sur les facteurs de risque, Bulletin Epidémiologique Hebdomadaire, $\mathrm{n}^{\circ}$ 5, 2015, pp. 76-82.

2 Ministère de l'écologie, du développement-durable des transports et du logement, Circulaire du 18 mai 2011 relative aux mesures exceptionnelles de limitation ou de suspension des usages de l'eau en période de sécheresse, Bulletin officiel, $\mathrm{n}^{\circ} 13,2011, \mathrm{p} .93$.

3 François Calvet, Christian Manable, Xynthia, 5 ans après : pour une véritable culture du risque dans les territoires, Rapport d’information du Sénat, n536, 2015, pp. 15-37. 
climatiques pour le $\mathrm{XXI}^{\mathrm{e}}$ siècle synthétisées dans le $5^{\mathrm{e}}$ rapport d'évaluation du Groupe intergouvernemental d'experts sur l'évolution du climat (GIEC). 
Au cours des années 1990, le développement des connaissances relatives au changement climatique a suscité la mise en place d'un nouveau cadre institutionnel au niveau international, européen, national et infra-national afin de définir et de piloter les politiques climatiques dans une perspective de développement durable. La présentation de l'évolution progressive du cadre institutionnel est l'occasion d'aborder avec un peu plus de détail le dernier élément mis en place en France: la politique publique d'adaptation au changement climatique.

Enfin, au travers d'exemples sectoriels relatifs aux infrastructures et d'exemples de territoires littoraux, la troisième partie identifie des besoins sociaux nouveaux liés à l'adaptation au changement climatique. Ce qui permet d'illustrer l'aspect moteur de l'adaptation au changement climatique pour l'innovation sociale dans une perspective de développement durable.

\section{LE CLIMAT CHANGE}

Le climat est la branche de la météorologie qui s'intéresse au temps long. L'Organisation météorologique mondiale (OMM) considère que pour représenter le climat d'un lieu donné en termes de paramètres moyens et de variabilité, plusieurs décennies sont nécessaires. La durée typiquement utilisée par les services météorologiques pour caractériser le climat d'une zone géographique est de 30 ans. Afin de présenter le contexte général de notre approche, cette première partie présente les grandes évolutions du climat à différentes échelles de temps long ainsi que les principaux déterminants de ces évolutions.

\section{À l'échelle paléoclimatique}

En exploitant ce qui est fréquemment qualifié d' «archives climatiques » (ex : bulles d'air emprisonnées dans les glaces, sédiments marins, récifs coralliens, cernes d'arbres) il est possible de reconstruire l'évolution de certains paramètres caractérisant le climat mondial sur une durée maximale de plusieurs millions d'années avec une précision plus grande pour les 800000 dernières années ${ }^{4}$. Au cours de cette période, plusieurs alternances de phases glaciaires et interglaciaires se sont succédées suivant des cycles d'approximativement $100 \quad 000$ à $120 \quad 000$ ans. La variation des paramètres astronomiques associés à la trajectoire de la terre autour du soleil (ex. : excentricité, précession et inclinaison) est le déterminant principal de ces cycles en modulant l'intensité du rayonnement solaire incident moyen à la surface de la terre.

Par exemple, au cours des derniers 800000 ans, le niveau moyen des mers a fluctué entre $+5 \mathrm{~m}$ à $+10 \mathrm{~m}$ (en période interglaciaire) et $-100 \mathrm{~m}$ à $-120 \mathrm{~m}$ (en période glaciaire) par rapport au niveau moyen du $\mathrm{XX}^{\mathrm{e}}$ siècle. La température moyenne de surface des océans tropicaux a fluctué entre $+1^{\circ} \mathrm{C}$ et $-4^{\circ} \mathrm{C}$ par rapport à la température moyenne du $\mathrm{XX}^{\mathrm{e}}$ siècle et celle de $1^{\prime}$ Antarctique a fluctué entre $+2^{\circ} \mathrm{C}$ et $-8^{\circ} \mathrm{C}$.

4 Intergovernmental Panel on Climate Change, Climate Change 2013: The Physical Science Basis. Contribution of Working Group I to the Fifth Assessment Report of the Intergovernmental Panel on Climate Change, Cambridge University Press, 2013, pp. 383-464. 
Aux latitudes tempérées, la différence entre une période glaciaire et une période interglaciaire se caractérise par une différence de température de surface de 4 à $6^{\circ} \mathrm{C}$. Ces variations se sont produites en plusieurs milliers d'années. La variation la plus rapide observée à la fin du dernier minimum glaciaire est de l'ordre $1^{\circ} \mathrm{C}$ en 1000 ans (en réchauffement comme en refroidissement).

Les variations dont il est question ont eu lieu pendant les époques les plus récentes de l'ère quaternaire (débutée il y a 2,6 millions d'années avec l'apparition de l'homme) pendant laquelle la position des continents peut être considérée comme proche de l'actuelle. Le dernier maximum interglaciaire a eu lieu il y a 120000 ans environ. La fin du dernier minimum glaciaire marquant le début de l'époque actuelle, nommée « holocène », est datée entre 10000 et 12000 ans avant aujourd'hui.

En parallèle des variations des paramètres météorologiques et océaniques, la concentration de dioxyde de carbone dans la troposphère a fluctué entre 180 et 300 parties par million (ppm) au cours des 800000 dernières années. Les concentrations les plus faibles sont observées durant les périodes les plus froides et les concentrations les plus élevées durant les périodes les plus chaudes ${ }^{5}$.

\section{À l'échelle de quelques siècles}

$\mathrm{Au}$ cours des 6000 dernières années, le niveau moyen des mers s'est élevé d'environ $2,5 \mathrm{~m}$ à un rythme de $5 \mathrm{~cm}$ par siècle entre -6000 et -3000 , ensuite la hausse s'est ralentie puis le niveau moyen des mers s'est stabilisé ${ }^{6}$. En effet, entre -2000 ans et le début du XIX ${ }^{\mathrm{e}}$ siècle, le niveau moyen des mers a fluctué seulement d'une dizaine de centimètres. À partir du milieu de $\mathrm{XIX}^{\mathrm{e}}$ siècle le niveau moyen des mers augmente à une vitesse entre 15 et $20 \mathrm{~cm}$ par siècle. Depuis la fin du $\mathrm{XX}^{\mathrm{e}}$ siècle, l'élévation s'effectue à un rythme de $30 \mathrm{~cm}$ par siècle. Les deux facteurs contributeurs majeurs à la hausse du niveau moyen des mers sont la fonte des glaces continentales et la dilatation des océans.

$\mathrm{Au}$ cours des 2000 dernières années, l'évolution de la température moyenne a connu également plusieurs fluctuations ${ }^{7}$ comme l'illustre, par exemple pour l'hémisphère nord, la période appelée «petit âge glaciaire » (1450-1800) dont la température moyenne était inférieure de 0,3 à $0,4^{\circ} \mathrm{C}$ à celle de la période appelée « optimum médiéval » (950-1250) qui l'a précédée. Depuis le milieu du XIX ${ }^{\mathrm{e}}$ siècle, les techniques et protocoles de mesure se sont perfectionnés et permettent désormais de

5 Ces évolutions traduisent la part atmosphérique des fluctuations du cycle du carbone.

6 Intergovernmental Panel on Climate Change, Climate Change 2013: The Physical Science Basis. Contribution of Working Group I to the Fifth Assessment Report of the Intergovernmental Panel on Climate Change, Cambridge University Press, 2013, pp. 255-316.

7 Intergovernmental Panel on Climate Change, Climate Change 2013: The Physical Science Basis. Contribution of Working Group I to the Fifth Assessment Report of the Intergovernmental Panel on Climate Change, Cambridge University Press, 2013, pp. 159-254. 
déterminer la part de l'activité solaire (cycles d'environ 11 ans), des grandes éruptions volcaniques (effet refroidissant pendant plusieurs années) et de l'influence des gaz à effet de serre d'origine anthropique (ex. : dioxyde de carbone, protoxyde d'azote, méthane, gaz fluorés $)^{8}$. La hausse de la température moyenne de la terre entre 1900 et 2010 est de $0,85^{\circ} \mathrm{C}$. Au fil des décennies, les ré-analyses des situations passées intégrant l'ensemble des éléments connus (paramètres astronomiques, activité solaire, éruptions volcaniques, thermodynamique atmosphérique et océanique) permettent de restituer l'évolution passée du climat à long terme ainsi que sa variabilité interne (petites échelles de temps) si et seulement si l'influence des gaz à effet de serre d'origine anthropique est incluse dans la représentation. En effet, si cette influence n'est pas prise en compte, une grande majorité des séries de mesures de température à la surface de la terre ne peut être reproduite.

Les effets les plus visibles du changement climatique s'observent aux plus hautes latitudes de l'hémisphère nord (diminution de l'épaisseur et de l'extension de la banquise arctique en été, diminution de la surface enneigée au printemps), dans les zones de montagne (recul des glaciers, surfaces enneigées au printemps) et les zones côtières (hausse du niveau marin) ${ }^{9}$.

Malgré les fluctuations majeures relevées à l'échelle de plusieurs millénaires, à la variabilité inter-annuelle près, le climat pouvait donc être considéré comme «stable » à l'échelle d'un siècle jusqu'au $\mathrm{XIX}^{\mathrm{e}}$ siècle. Mais depuis le milieu du XX $\mathrm{X}^{\mathrm{e}}$ siècle, le climat est en transition vers un nouvel état d'équilibre, rapide par rapport à ce qui a été observé au cours des siècles précédents. Cet état d'équilibre dépendra du niveau de stabilisation des gaz à effet de serre anthropiques dans l'atmosphère.

\section{Évolutions futures}

L'évolution future du climat dépend de plusieurs facteurs dont certains peuvent être anticipés de manière relativement robuste comme les paramètres astronomiques. D'autres, comme les grandes éruptions volcaniques, ne peuvent pas être anticipés du tout. Pour ce qui est de l'influence des gaz à effet de serre, les contributions peuvent être anticipées de différentes manières. Le premier élément à noter est que l'effet

8 L'eau sous forme de vapeur est un gaz à effet de serre naturel très abondant dans l'atmosphère. La vapeur d'eau contribue à l'effet de serre naturel qui permet à la température moyenne en surface d'atteindre $15^{\circ} \mathrm{C}$ au lieu d'environ $-18^{\circ} \mathrm{C}$ s'il n'agissait pas.

9 Intergovernmental Panel on Climate Change, Climate Change 2014: Impacts, Adaptation and Vulnerability. Contribution of Working Group II to the Fifth Assessment Report of the Intergovernmental Panel on Climate Change, Cambridge University Press, 2014, pp. 986-995. 
cumulé des gaz à effet de serre ${ }^{10}$ déjà émis a conduit à un déséquilibre radiatif ${ }^{11} \mathrm{du}$ système terre, océan, atmosphère, cryosphère, biosphère. L'atteinte d'un nouvel état d'équilibre du système est estimée à plusieurs décennies après une stabilisation de la concentration des gaz à effet de serre dans l'atmosphère. De plus, la trajectoire des émissions mondiales de gaz à effet de serre ne montre pas d'inflexion actuellement (au moins jusqu'en 2012) d'une part et la plupart des systèmes économiques sont basés sur des techniques à forte intensité en carbone d'autre part. La démographie mondiale, la structure des systèmes économiques combinés à l'inertie du système climatique ne laissent pas entrevoir de diminution tendancielle de la concentration de gaz à effet de serre à court terme. C'est précisément pour cette raison qu'il était nécessaire de parvenir à un accord multilatéral ambitieux porté au plus au niveau des Etats lors de la $21^{\mathrm{e}}$ conférence des parties (COP21) à la Convention cadre des Nations-unies sur les changement climatiques.

Les projections réalisées par les climatologues en utilisant les modèles du système terre tiennent compte de différentes possibilités d'évolution de la société (ex. : usage de combustibles fossiles, occupation des sols) de manière intégrée par l'intermédiaire du déséquilibre radiatif résultant. La famille de projections climatiques associées aux plus faibles trajectoires d'émission de gaz à effet de serre réalisées dans le cadre du $5^{\mathrm{e}}$ rapport d'évaluation du GIEC est nommée RCP2.6 (Representative Concentration Pathway $2.6 \mathrm{~W} / \mathrm{m}^{2}$ ). Il s'agit de scénarios compatibles avec la stabilisation de la concentration de gaz à effet de serre anthropique dans l'atmosphère (ex. : moins de 450 ppm en 2100). Ceci revient à infléchir puis réduire rapidement (avant 2030) les émissions de gaz à effet de serre. La famille de projections climatiques associées aux plus fortes émissions est nommée RCP8.5. Il s'agit d'une forme de scénario tendanciel par rapport aux émissions de gaz à effet de serre relevées au cours de la dernière décennie. L'ensemble des 39 modèles climatiques analysés par le GIEC pour la famille RCP8.5 conduit à un niveau de réchauffement en moyenne annuelle mondiale de l'ordre de $4^{\circ} \mathrm{C}$ pour la période 2081-2100 par rapport à la période 1985-2005. L'ensemble des 32 modèles climatiques analysés par le GIEC pour la famille RCP2.6 conduit à un niveau de réchauffement en moyenne annuelle mondiale de 1 'ordre de $1^{\circ} \mathrm{C}$ pour la période 2081-2100 par rapport à la période $1985-2005^{12}$. Concernant le niveau moyen des mers, l'évolution projetée se situe entre $+26 \mathrm{~cm}$ et $+82 \mathrm{~cm}$ sur la même période.

10 En 2012, la concentration de gaz à effet de serre atteint 450 ppm d'équivalent $\mathrm{CO}_{2}$, ce qui représente 1,5 fois le maximum relevé au cours des 800000 dernières années.

11 De l'ordre de 2 à $3 \mathrm{~W} / \mathrm{m}^{2}$ pour un flux incident net de $240 \mathrm{~W} / \mathrm{m}^{2}$ au sommet de l'atmosphère.

12 Intergovernmental Panel on Climate Change, Climate Change 2013: The Physical Science Basis. Contribution of Working Group I to the Fifth Assessment Report of the 
Ainsi, quelles que soient les familles de scénario considérées, si les variations projetées de la température moyenne pour un siècle sont de l'ordre de grandeur de variations déjà observées, les durées associées par le passé étaient 10 à 100 fois plus longues.

\section{LE CADRE INSTITUTIONNEL EVOLUE}

Compte tenu de l'analyse précédente, il est apparu de plus en plus nettement depuis le début des années 1990 que la question du changement climatique est à aborder sur deux plans : celui de l'atténuation des émissions de gaz à effet de serre et celui de l'adaptation des sociétés aux effets du changement climatique. Ces deux aspects sont liés, puisque les besoins d'adaptation seront d'autant plus limités que le niveau de stabilisation de la concentration de gaz à effet de serre d'origine anthropique dans l'atmosphère sera faible. Inversement, des efforts d'adaptation qui augmenteraient les émissions de gaz à effet de serre, contribueraient à amplifier les évolutions du climat auxquelles il s'agit de faire face. Ces deux aspects sont donc indissociables dans une perspective de long terme (plusieurs décennies).

La période actuelle présente deux caractéristiques inédites importantes pour la société. La première caractéristique est la conscience de l'évolution du climat à différentes échelles de temps et de l'amplitude des variations de certains paramètres. La seconde caractéristique est la capacité à objectiver des futurs possibles pour le climat mondial et ses déclinaisons locales. Ces deux caractéristiques, rendues possibles à la fois par des avancées conceptuelles, le développement des moyens de calcul et la collaboration internationale d'équipes de recherche, sont inédites à l'échelle de l'histoire humaine.

Contrairement à la fin des années 1990, la question n'est plus de savoir si la société doit s'adapter ou non aux conditions climatiques projetées pour le milieu ou la fin du siècle, mais bien d'imaginer comment s'adapter au changement climatique et quels objectifs rechercher.

\section{Développement des politiques climatiques}

Le développement des connaissances relatives à l'évolution du climat puis à l'influence des activités humaines sur cette évolution sont devenues suffisantes pour mobiliser la communauté internationale au plus haut niveau lors du «Sommet de la Terre » organisé à Rio en 1992. Avant cette date, le changement climatique était principalement un sujet abordé dans la communauté mondiale de recherche en sciences du climat, fédérée autour du programme mondial de recherche sur le climat mis en place par l'OMM à la fin des années 1970. La première synthèse mondiale des connaissances sur le climat, destinée à diffuser l'information au-delà de la communauté

Intergovernmental Panel on Climate Change, Cambridge University Press, 2013, pp. 10291136. 
de recherche, a été réalisée par le GIEC dans son premier rapport d'évaluation publié en 1991.

A Rio, les chefs d'État et de gouvernement se sont accordés pour limiter l'influence des activités humaines sur le climat à un niveau ne présentant pas de dangers pour la production alimentaire, le développement économique et l'adaptation naturelle des écosystèmes en adoptant la Convention cadre des nations unies pour les changements climatiques $(\mathrm{CCNUCC})^{13}$. Ce traité, signé par 154 pays à l'origine, et désormais ratifié par 195 États, matérialise le début du développement des politiques climatiques. Ces politiques se sont mises en place dans quatre directions complémentaires: deux axes internationaux (échelle mondiale) et deux axes domestiques (échelle nationale) ${ }^{14}$.

Au niveau domestique, il s'agit de l'atténuation des émissions de gaz à effet de serre pour limiter l'influence humaine sur l'évolution du climat, et de l'adaptation visant à limiter l'impact des effets néfastes et à saisir les opportunités du changement climatique. Au niveau international, il s'agit d'instituer les négociations climatiques mondiales traitant des aspects d'atténuation et d'adaptation d'une part et d'incorporer l'atténuation et l'adaptation dans les dispositifs d'aide au développement d'autre part.

Au cours des années 1990 la limitation des émissions de gaz à effet de serre a concentré la plupart des attentions et a abouti, à l'échelle mondiale, dans le cadre de la CCNUCC, à l'élaboration du protocole de Kyoto en 1997, signé par 83 États. En France, la mission interministérielle de l'effet de serre a été créée et placée auprès du premier Ministre en 1992, afin de coordonner l'action de la France dans sa lutte contre les émissions de gaz à effet de serre. Dissoute en 2002, ses missions sont désormais réparties au sein de différentes unités du Ministère chargé du développement durable (Service climat et efficacité énergétique et Direction des affaires européennes et internationales ${ }^{15}$ ) et du Ministère chargé des affaires étrangères. Sur une initiative parlementaire, le Sénat et l'Assemblée nationale adoptent à l'unanimité la loi du 17 février $2001^{16}$ qui, par son article $1^{\text {er }}$, confère à la lutte contre l'intensification de l'effet de serre et la prévention des risques liés au réchauffement climatique le caractère de priorité nationale. Cet acte législatif, inédit au niveau mondial, complète le dispositif

13 Nations Unies, Convention cadre des nations Unies sur les changements climatiques, 1992, p. 5.

14 Gaël Virlouvet, Vingt ans de lutte contre le réchauffement climatique en France : bilan et perspectives des politiques publiques, Conseil économique social et environnemental, 2015, pp. 82-92.

15 Respectivement rattachés de la Direction générale de l'énergie et du climat et au sein de Secrétariat général. 
national en identifiant explicitement le besoin d'action publique pour faire face aux effets du changement climatique. Pour ce faire, les articles suivants créent l'Observatoire national sur les effets du réchauffement climatique (Onerc) en lui attribuant les missions de collecte et de diffusion d'informations et de formulation de recommandations en matière d'adaptation à destination $\mathrm{du}$ gouvernement et du parlement. Placé initialement auprès du Premier ministre, en 2008 l'Onerc est rattaché à la Direction générale de l'énergie et du climat au sein du Ministère chargé du développement durable.

Ainsi se matérialise l'engagement de la démarche d'adaptation en France en tant que politique publique ${ }^{17}$. Avant de nous concentrer sur certains de ses aspects, il convient de noter que l'adaptation traite de l'effet de l'environnement sur les sociétés. Alors que l'atténuation traite de l'effet de la société sur l'environnement. Cette symétrie conduit à un changement de point de vue lorsqu'on passe de l'une à l'autre de ces politiques publiques complémentaires. L'articulation de ces deux points de vue correspond à aux politiques de développement bas carbone résilientes au changement climatique.

\section{Adaptation aux effets du changement climatique}

Les trajectoires de développement peuvent tenir compte, de différentes manières, des facteurs sociaux et environnementaux dans une perspective de développement durable. En ce qui concerne l'influence du climat sur la société, les aménagements humains existants sont, par exemple, plus ou moins résilients à la variabilité climatique présente. L'objectif de l'adaptation au changement climatique est de faire en sorte que la société future soit moins vulnérable à la variabilité climatique future que ne l'est la société actuelle à la variabilité climatique actuelle. De manière plus modeste, en prenant en compte la croissance démographique et la croissance économique, l'objectif pourrait être, dans un premier temps, de faire en sorte, pour un territoire donné, de renforcer la résilience plus rapidement que ne croissent les enjeux. Autrement dit, que la vulnérabilité relative diminue (ex. : réduction de la proportion de population exposée à un risque de submersion marine sévère) même si la vulnérabilité absolue augmente (ex. : augmentation du nombre d'habitants exposés à un risque de submersion marine sévère).

Une trajectoire de développement qui ne tient pas compte des futurs possibles du climat pour les éléments de long terme (plusieurs décennies) s'expose à ne pas considérer certaines vulnérabilités ou opportunités. Bien évidemment, le climat n'est pas le seul paramètre à intégrer dans le cadre d'une démarche de développement durable. De même, les dimensions d'une démarche de développement durable ne sont pas toutes sensibles au climat et à ses variations.

17 Observatoire national sur les effets du réchauffement climatique, L'adaptation de la France au changement climatique, Rapport au Premier ministre et au Parlement, La documentation Française, 2012, pp. 15-28. 
Un peu moins de six ans après le début de la démarche d'adaptation en France, la stratégie nationale est adoptée, fin 2006, en réunion interministérielle. Elle met en avant quatre finalités : protéger les personnes et les biens en agissant pour la sécurité et la santé publique, tenir compte des aspects sociaux et éviter les inégalités devant les risques, limiter les coûts et tirer parti des avantages et enfin, préserver le patrimoine naturel $^{18}$.

En 2009, une phrase de la loi de mise en œuvre du grenelle environnement indique que «la France se dotera avant fin 2011 d'un plan national d'adaptation au changement climatique ». Ainsi après un travail préliminaire d'estimation de coûts des impacts du changement climatique pour quelques activités et secteurs clés (ex. : santé publique, aménagement littoral, infrastructures) conduit par une équipe de recherche pluridisciplinaire, est mis en place un dispositif partenarial d'élaboration du premier plan national d'adaptation au changement climatique.

Le premier plan national d'adaptation, publié en 2011, est constitué d'un préambule rassemblant des éléments de diagnostic, une mise en perspective de la stratégie nationale d'adaptation puis de l'ensemble des 83 actions déclinées en 230 mesures ${ }^{19}$ regroupées au sein de 20 fiches thématiques ${ }^{20}$. Il s'agit d'un plan interministériel dont le principe général repose sur l'incorporation de l'adaptation au changement climatique au sein d'autres politiques publiques. L'élaboration comme le pilotage global du plan relève de la responsabilité de la Direction générale de l'énergie et du climat au sein du Ministère chargé du développement durable et la mise en œuvre est confiée, en fonction des thèmes, aux directions générales des ministères en charge de chaque sujet.

\section{L'ADAPTATION AU CHANGEMENT CLIMATIQUE LEVIER D'INNOVATION SOCIALE ?}

Les évolutions climatiques passées ont permis à l'homme l'accès à de nouveaux espaces devenus moins hostiles (ex. : moins froids ou moins arides). Selon certaines approches, l'évolution du climat est présentée comme un des facteurs déclencheurs potentiel de grandes migrations humaines s'étendant sur plusieurs générations ${ }^{21}$. Notre

18 Observatoire national sur les effets du réchauffement climatique, Stratégie nationale d'adaptation au changement climatique, Rapport au Premier ministre et au Parlement, La documentation Française, 2007, pp. 23-28.

19 Direction générale de l'énergie et du climat, Plan national d'adaptation au changement climatique 2011-2015, Ministère de l'écologie, du développement durable et de l'énergie, 2011, pp. 67-178.

20 Actions transversales, santé, biodiversité, recherche, littoral, prévention des risques, infrastructures et systèmes de transport, urbanisme et cadre bâti, montagne, tourisme, industrie et énergie, financement et assurance, information communication, pêche, agriculture, forêt, ressource en eau, éducation et formation, action européenne et internationale, gouvernance.

21 Robert Potts, Environmental hypothesis of hominid evolution, Yearbook of Physical Anthropology, n41, 1998, pp. 93-136. 
propos ne se situe par sur le plan des migrations humaines mais se focalise sur le territoire français et à l'échelle d'une ou deux générations.

Les projets individuels ou collectifs, s'ils ont vocation à se prolonger dans le temps, au-delà de quelques décennies, ne peuvent pas s'affranchir d'une confrontation aux futurs du climat ${ }^{22}$. En effet, un projet conçu pour la société de demain utilisant le climat d'hier comme référence a peu de chance d'être durable selon cette dimension.

\section{Illustrations sectorielles}

Il ne s'agit pas ici d'identifier toutes les vulnérabilités de la société au changement climatique, mais d'en exposer certaines pour illustrer les facettes variées de l'adaptation.

Dans un souci de limiter les besoins de chauffage, la pratique habituelle de construction se concentre sur l'isolation contre le froid extérieur et la conservation de la chaleur à l'intérieur des logements. Avec l'occurrence récente de plusieurs vagues de chaleurs intenses sur l'ensemble de la métropole, la question de confort d'été des bâtiments (logements, bureaux, ateliers, ...) commence à devenir un sujet de préoccupation important au-delà du tiers sud de la métropole et des outre-mer tropicaux. En effet, l'enjeu est d'éviter la conservation et le stockage de chaleur en période chaude ou de favoriser la conservation du froid dans les bâtiments. D'un point de vue des particuliers, il s'agit de limiter l'exposition des populations à des chaleurs excessives dans leurs logements. Du point de vue des professionnels, il s'agit d'éviter l'exposition des travailleurs ${ }^{23}$ et des matériels à des conditions pénalisant la productivité des processus. À l'échelle des bâtiments, ces enjeux peuvent être traités par exemple au niveau de l'isolation, du choix des matériaux, de l'orientation des façades ou de l'occultation des ouvertures ${ }^{24}$. À l'échelle d'un quartier ou d'une ville, l'action peut se situer au niveau de l'implantation des espaces verts, de l'espacement des bâtiments, l'orientation des rues ${ }^{25}$, notamment pour limiter au maximum l'effet d'îlot de chaleur

22 Antoine Bonduelle, Jean Jouzel, L'adaptation de la France au changement climatique mondial, Conseil économique social et environnemental, 2014, pp. 23-32.

23 Catherine Buisson, Impact sanitaire de la vague de chaleur de l'été 2006 en milieu de travail, Institut de veille sanitaire, 2009, pp. 8-10.

24 Sylviane Roger, Michel Carré, Améliorer le confort d'été dans les établissements pour personnes âgées et handicapées, Cahier Pratique, Direction générale de l'action sociale et Agence de l'environnement et de la maîtrise de l'énergie, 2009, pp. 26-42.

25 Observatoire national sur les effets du réchauffement climatique, Ville et adaptation au changement climatique, Rapport au Premier ministre et au Parlement, La documentation française, 2010, pp. 67-69. 
urbain $^{26}$. Au niveau institutionnel il s'agit par exemple d'identifier les personnes les plus vulnérables aux fortes chaleurs, d'organiser les services de santé publique, de mettre en alerte des services d'urgence, d'informer les populations, d'identifier des lieux rafraîchis pour l'accueil du public ${ }^{27}$. Certains de ces éléments ne sont pas nouveaux, mais la plupart ne sont que partiellement pris en compte dans les projets.

Le recours à la climatisation individuelle est un cas intéressant de transfert de vulnérabilité. En effet, s'il a un effet bénéfique immédiat et direct sur le logement équipé, il présente un certain nombre de limitations. En particulier, un recours massif à la climatisation individuelle renforce l'effet d'îlot de chaleur urbain à l'échelle d'un quartier et aggrave donc la situation des logements non équipés. Rafraîchir de quelques degrés au lieu de rechercher le maintien d'une température idéale beaucoup plus basse suffit pourtant à réduire significativement la vulnérabilité des personnes. Sachant que l'augmentation significative du nombre de jours de forte chaleur est une des conclusions les plus robustes des projections climatiques ${ }^{28}$, le développement urbain peut-il prétendre être durable s'il n'intègre pas tous ces enjeux dans son approche ?

D'autres illustrations concernant les infrastructures et le cadre bâti sont aisées à appréhender. L'augmentation de la température moyenne de l'air conjuguée à l'occurrence plus fréquente de jours de forte chaleur produit un assèchement des sols superficiels très important. La sécheresse des sols amplifie le phénomène de retrait gonflement des sols argileux. Ce phénomène de dilatation-rétractation en fonction de l'humidité des sols peut produire des fissures dans les façades lorsque les fondations sont dimensionnées au plus juste par rapport au comportement standard du sol. Pour ce qui est des systèmes de transport, la dilatation et la déformation des rails de chemin de fer ou le stockage des wagons au soleil rendent les voies et les rames inutilisables en période de forte chaleur.

Les effets du changement climatique peuvent également induire des phénomènes de «désadaptation ». Par exemple, dans le cas de gestion de la viabilité hivernale des infrastructures, l'absence récurrente de fortes chutes de neige peut conduire à ne pas renouveler le matériel de déneigement et donc à induire une vulnérabilité nouvelle en cas d'épisode dont l'occurrence, même plus espacée, reste possible. Les gestionnaires

26 Température excédentaire en zone urbanisée dense par rapport à la périphérie. Les cas extrêmes en période de forte chaleur peuvent dépasser $10^{\circ} \mathrm{C}$ d'écart, en fin de nuit, entre le centre-ville et la périphérie rurale.

27 Observatoire national sur les effets du réchauffement climatique, Changement climatique et risques sanitaires en France, Rapport au Premier ministre et au Parlement, La documentation française, 2008, pp. 119-127.

28 Gaëlle Ouzeau, Michel Déqué, Manel Jouini, Serge Planton, Robert Vautard, Le climat de la France au XXI siècle : Projection climatiques régionalisées, Ministère de l'écologie, du développement-durable et de l'énergie, volume 4, 2014, pp. 3-4. 
d'infrastructures sont ainsi confrontés à des sujets inédits difficiles à traiter dans le cadre d'approches habituelles. Quel sens donner à la fréquence d'occurrence d'un événement météorologique dans le contexte d'un climat non stationnaire ? Ou encore, comment tenir compte de cette information en matière de conception et de gestion? La question de la durée de retour d'un événement en climat non stationnaire et du coût des conséquences associées revêt un caractère particulièrement critique pour le secteur des assurances. Ces constats ont conduit l'Union européenne a mettre en avant la question des référentiels au travers l'examen des «Eurocodes » et des systèmes d'assurance et de ré-assurance des État membres ${ }^{29}$. Le modèle économique de ce secteur est-il durable dans un contexte de changement climatique?

Les sciences du climat enrichissent les connaissances sur ces questions notamment sur le thème de la détection-attribution du changement climatique en visant, à moyen terme le développement de méthodologies nouvelles pour la caractérisation des phénomènes et de leurs effets. Parallèlement, certaines organisations professionnelles, notamment dans le domaine des infrastructures, examinent les normes actuelles pour identifier leur sensibilité aux évolutions climat et identifier les premières pistes d'évolution des référentiels ${ }^{30}$.

La plupart des éléments de vulnérabilité précédents ont été rendus particulièrement visibles à l'occasion de la canicule qui a touché l'Europe de l'ouest et principalement la France en 2003. Cela a mis en évidence l'inadaptation de nos référentiels de construction et d'urbanisme à la variabilité actuelle du climat. Cette inadaptation préfigure certaines de nos vulnérabilités pour des futurs plus chauds du climat métropolitain. Quant aux opportunités, elles n'apparaissent pas toutes de manière aussi évidentes car il ne s'agit pas de dégradation d'une situation connue mais d'imaginer ce qui pourrait être fait pour tirer parti d'une situation différente. À l'échelle d'un continent, il peut s'agir de l'évolution de l'aire de culture du blé par exemple ${ }^{31}$.

29 Commission-Européenne, Stratégie de l'UE relative à l'adaptation au changement climatique, Direction générale de l'action climatique, Communication de la commission au parlement européen, au conseil, au comité économique et social européen et au comité des régions, 2013, pp. 10-11.

30 Direction technique infrastructures de transport et matériaux, Plan National d'Adaptation au Changement Climatique, volet infrastructures et systèmes de transport, action 1 Impacts potentiels du changement climatique sur les infrastructures et systèmes de transport, sur leurs référentiels de conception, entretien et exploitation, et besoins de précisions des projections climatique, Centre d'étude et d'expertise sur les risques, l'environnement, la mobilité et l'aménagement, 2015, 157 p.

31 Intergovernmental Panel on Climate Change, Climate Change 2014: Impacts, Adaptation and Vulnerability. Contribution of Working Group II to the Fifth Assessment Report 


\section{Projets de territoires}

En considérant l'aménagement littoral, il est possible d'illustrer de manière spectaculaire l'évolution de nos vulnérabilités. En effet, depuis le milieu du XX $\mathrm{X}^{\mathrm{e}}$ siècle, de très nombreux aménagements côtiers ont été réalisés en France accompagnant le goût des populations pour l'espace littoral. Confrontés à une triple problématique selon des échelles de temps différentes, de flux migratoires, d'érosion côtière et de hausse du niveau marin, les collectivités littorales illustrent la nécessité de conduire une réflexion à long terme intégrant de nombreuses dimensions ${ }^{32}$. En effet, au regard des événements de submersions survenus au cours des dernières décennies, la pérennité de certains aménagements côtiers n'aura pas dépassé 50 ans. De plus, certaines collectivités sont fortement endettées par l'accumulation des travaux récurrents de défense contre l'érosion ou la submersion marine et ne pourront plus assumer le mode de gestion selon un cycle protection-destruction-reconstruction. Dans certains cas, il s'agit déjà de situation de crise, dans d'autre le temps nécessaire pour le développement d'action de prévention est encore disponible. Cependant, dans tous les cas, l'imbrication des intérêts et des responsabilités, la nature variée des enjeux avérés ou potentiels conduit à des situations complexes dont la gestion n'est pas à la portée des pratiques courantes. Les tensions révélées lors de l'événement Xynthia ainsi que pendant les développements judiciaires ultérieurs en sont une manifestation ${ }^{33}$. Dans un contexte d'accélération de la hausse du niveau marin, se posent, de manière plus sensible et parfois urgente, les questions de : relocalisation des biens, disponibilité foncière rétro-littorale, perte de patrimoine (matériel ou immatériel) des particuliers et de la collectivité, protection des populations, valorisation du linéaire côtier, développement de l'activité économique, la durée de retour sur investissement des investissements.

La situation actuelle traduit le fait que l'adaptation spontanée des populations n'est pas suffisante, les outils de planification (ex : Plan de prévention des risques naturels littoraux, Plan local d'urbanisme, Schéma de cohérence territorial) peuvent répondre aux besoins les plus critiques à court et moyen termes. Cependant, une approche intégrative à l'échelle du risque considérée reste à imaginer, territoire par territoire, pour traiter durablement ces questions ${ }^{34}$. Dans le but de favoriser l'émergence de ce type d'approche, l'État finance dans le cadre de la mise en œuvre de la stratégie nationale de gestion intégrée du trait de côte, et du développement des plans de

of the Intergovernmental Panel on Climate Change, Cambridge University Press, 2014, pp. 986-995.

32 Observatoire national sur les effets du réchauffement climatique, Littoral et changement climatique : Rapport au Premier ministre et au Parlement, La documentation française, 2015 (à paraître).

33 Procès Xynthia : quatre et deux ans de prison pour l'ancien maire et son adjointe, «Le Parisien » du 12 décembre 2014.

34 Commissariat général à l'égalité des territoires, Synthèse des études inter-régionales sur l'adaptation au changement climatique, 2015 (à paraitre). 
prévention des risques naturels côtiers et du plan national d'adaptation au changement climatique, des travaux de recherche traitant d'aménagement côtier portés par des collectivités littorales ${ }^{35}$. L'objectif de cette démarche est notamment d'identifier les principaux enjeux à prendre en compte et des pistes de traitement de ces enjeux malgré des opinions parfois contradictoires. Partant du constat des limites de la gestion actuelle, l'État a donc financé cette démarche, sans doctrine pré-établie, d'appui de cinq collectivités territoriales $^{36}$ avec des problématiques variées dans une optique d'innovation sociale face à un sujet dont le traitement fin ne peut être qu'envisager localement. L'analyse de ces travaux permettra potentiellement d'identifier des innovations pour faire face à des problématiques nouvelles en s'appuyant sur les connaissances les plus avancées.

La question de l'aménagement littoral est en lumière aujourd'hui probablement pour deux raisons principales : la forte visibilité des phénomènes de submersion marine souvent spectaculaire marquant ainsi les esprits et la tendance à concentrer des enjeux patrimoniaux dans l'espace littoral. Les territoires insulaires dont une forte proportion peut être considérée comme du domaine littoral concentrent les problématiques à traiter $^{37}$. Les territoires de montagne présentent également de fortes vulnérabilités. En effet, un écart de température de $1^{\circ} \mathrm{C}$ correspond à une variation d'altitude d'environ $150 \mathrm{~m}$. Le changement climatique a donc un impact important sur l'étagement des peuplements végétaux, des pratiques agricoles, la gestion des routes ou la viabilité du tourisme de neige à basse altitude ${ }^{38}$. Suivant la même logique que pour le littoral, des projets de recherche pluridisciplinaires (ex. projet Adamont) s'engagent avec des territoires de montagne pour concevoir des pistes d'actions appropriées aux nouveaux défis à relever.

35 Direction générale de l'aménagement, du logement et de la nature, Vers la relocalisation des activités et des biens 5 territoires en expérimentation, Actes du séminaire à mi-parcours du 19 mai 2014, Ministère de l'écologie, du développement durable et de l'énergie, 2014, 64p.

36 Hyères dans le Var, Lacanau et la Teste de Buch en Aquitaine, Ault en Picardie, PetitBourg en Guadeloupe, Vias dans l'Hérault.

37 Observatoire national sur les effets du réchauffement climatique, Rapport au Premier ministre et au Parlement : Les outre-mer face au défi du changement climatique, La documentation française, 2013, pp. 175-182.

38 Observatoire national sur les effets du réchauffement climatique, Coût des impacts et pistes d'adaptation, Rapport au Premier ministre et au Parlement, La documentation française, 2009, pp. 109-111. 


\section{CONCLUSION}

Compte tenu des effets déjà observés du changement climatique et des effets supplémentaires inévitables en raison de l'inertie du système climatique, l'adaptation au changement climatique est un sujet dont le traitement ne peut plus être différé. L'adaptation au changement climatique contribue à l'émergence de besoins sociaux nouveaux et à l'émergence de traitement différent de besoins sociaux connus. Ces besoins émergent aussi bien au niveau individuel qu'au niveau collectif. Le dispositif d'adaptation en cours de développement au niveau de l'État ne vise pas à répondre à l'ensemble des besoins, mais plutôt à créer les conditions d'innovation qui permettront de faire face aux nouveaux défis. Il s'agit en particulier de promouvoir une approche intégrative conduisant à de véritables stratégies de développement et des projets de territoire permettent de prendre en compte la complexité du sujet dans toutes ses dimensions et d'orienter les pratiques vers une trajectoire de développement durable dans un contexte de changement climatique. Cette approche est en cohérence avec les orientations contenues dans l'accord de Paris sur le climat conclut en 2015 lors de la COP2 1 et les premiers éléments de sa mise en œuvre décidés à la $22^{\mathrm{e}}$ conférence des parties réunie à Marrakech en novembre 2016.

Aussi, l'innovation en matière de solutions techniques, de méthode de réflexion, d'identification d'opportunités, de modes de gouvernance sont des pistes dont le changement climatique facilite l'exploration. Au travers de projets de territoires, de projets d'entreprises, de projets individuels, l'adaptation au changement climatique constitue donc un potentiel levier d'innovation sociale au service du développement durable.

Plus généralement, le changement climatique contribue à interroger les méthodes employées en matière d'orientation du développement en révélant certaines vulnérabilités sociales créées au fil du temps. La non prise en compte de facteurs climatiques et l'optimisation des choix sur la base de référentiels climatiques passés a conduit à la création de vulnérabilités. Aussi, il est légitime de se demander si l'adaptation au changement climatique est compatible avec l'optimisation par rapport à des référentiels futurs. Ne serait-il pas plus judicieux d'envisager l'adaptation au changement climatique comme l'aménagement, ou le réaménagement, d'un espace de flexibilité dans une trajectoire de développement en intégrant notamment les futurs du climat?

\section{REFERENCES BIBLIOGRAPHIQUES}

Bonduelle (Antoine), Jouzel (Jean), L'adaptation de la France au changement climatique mondial, Conseil économique social et environnemental, 2014, 88 p.

Buisson Catherine, Impact sanitaire de la vague de chaleur de l'été 2006 en milieu de travail, Institut de veille sanitaire, 2009, 20 p.

Code de l'environnement, Partie législative, Livre II : Milieux physiques, Titre II : Air et atmosphère, Chapitre IX : Effet de serre. 
Commissariat général à l'égalité des territoires, Synthèse des études inter-régionales sur l'adaptation au changement climatique, 2015 (à paraître).

Commission-Européenne, Stratégie de l'UE relative à l'adaptation au changement climatique, Direction générale de l'action climatique, Communication de la commission au parlement européen, au conseil, au comité économique et social européen et au comoté des régions, 2013, 13 p.

Direction générale de l'aménagement, du logement et de la nature, Vers la relocalisation des activités et des biens 5 territoires en expérimentation, Actes du séminaire à mi-parcours du 19 mai 2014, Ministère de l'écologie, du développement durable et de l'énergie, 2014, 64p.

Direction générale de l'énergie et du climat, Plan national d'adaptation au changement climatique 2011-2015, Ministère de l'écologie, du développement durable et de l'énergie, 2011, $186 \mathrm{p}$.

Direction technique infrastructures de transport et matériaux, Plan National d'Adaptation au Changement Climatique, volet infrastructures et systèmes de transport, action 1 Impacts potentiels du changement climatique sur les infrastructures et systèmes de transport, sur leurs référentiels de conception, entretien et exploitation, et besoins de précisions des projections climatique, Centre d'étude et d'expertise sur les risques, l'environnement, la mobilité et l'aménagement, 2015, 157 p.

Calvet (François), Manable (Christian), Xynthia, 5 ans après : pour une véritable culture du risque dans les territoires, Rapport d'information du Sénat, n536, 2015, $94 \mathrm{p}$.

Laaidi (Karine), Ung (Aymeric), Pascal (Mathilde), Beaudeau (Pascal), «Vulnérabilité à la chaleur : actualisation des connaissances sur les facteurs de risque », Bulletin Epidémiologique Hebdomadaire, n5, 2015, pp. 76-82.

Intergovernmental Panel on Climate Change, Climate Change 2013: The Physical Science Basis. Contribution of Working Group I to the Fifth Assessment Report of the Intergovernmental Panel on Climate Change, Cambridge University Press, 1535 p.

Intergovernmental Panel on Climate Change, Climate Change 2014: Impacts, Adaptation and Vulnerability. Part A : Global and Sectoral Aspects, Contribution of Working Group II to the Fifth Assessment Report of the Intergovernmental Panel on Climate Change, Cambridge University Press, 1132 p.

Ministère de l'écologie, du développement-durable des transports et du logement, «Circulaire du 18 mai 2011 relative aux mesures exceptionnelles de limitation ou de suspension des usages de l'eau en période de sécheresse », Bulletin officiel, n ${ }^{\circ}$, 2011, pp. 92-113.

Nations Unies, Convention cadre des nations Unies sur les changements climatiques, 1992, 25p. 
Observatoire national sur les effets du réchauffement climatique, Coût des impacts et pistes d'adaptation, Rapport au Premier ministre et au Parlement, La documentation française, 2009, $194 \mathrm{p}$.

Observatoire national sur les effets du réchauffement climatique, L'adaptation de la France au changement climatique, Rapport au Premier ministre et au Parlement, La documentation Française, 2012, 125p.

Observatoire national sur les effets du réchauffement climatique, Les outre-mer face au défi du changement climatique, Rapport au Premier ministre et au Parlement, La documentation française, 2013, $214 \mathrm{p}$.

Observatoire national sur les effets du réchauffement climatique, Littoral et changement climatique: Rapport au Premier ministre et au Parlement, La documentation française, 2015 (à paraître).

Observatoire national sur les effets du réchauffement climatique, Changement climatique et risques sanitaires en France, Rapport au Premier ministre et au Parlement, La documentation française, 2008, 208 p.

Observatoire national sur les effets du réchauffement climatique, Stratégie nationale d'adaptation au changement climatique, Rapport au Premier ministre et au Parlement, La documentation Française, 2007, 96 p.

Observatoire national sur les effets du réchauffement climatique, Ville et adaptation au changement climatique, Rapport au Premier ministre et au Parlement, La documentation française, 2010, $224 \mathrm{p}$.

Ouzeau (Gaëlle), Déqué (Michel), Jouini (Manel), Planton (Serge), Vautard (Robert), Le climat de la France au XXI siècle : Projection climatiques régionalisées, Ministère de l'écologie, du développement-durable et de l'énergie, volume 4, 2014, 62 p.

Potts (Robert), «Environmental hypothesis of hominid evolution », Yearbook of Physical Anthropology, n41, 1998, pp. 93-136.

Roger (Sylviane), Carré (Michel), Améliorer le confort d'été dans les établissements pour personnes âgées et handicapées, Cahier Pratique, pour les gestionnaires d'établissement sociaux et médicaux sociaux, Direction générale de l'action sociale et Agence de l'environnement et de la maîtrise de l'énergie, 38 p.

Virlouvet (Gaël), Vingt ans de lutte contre le réchauffement climatique en France : bilan et perspectives des politiques publiques, Conseil économique social et environnemental, 2015, $298 \mathrm{p}$. 